\title{
PRODUCTS OF UNBOUNDED BLOCH FUNCTIONS
}

\author{
DANIEL GIRELA
}

\begin{abstract}
We give new constructions of pair of functions $(f, g)$, analytic in the unit disc, with $g \in H^{\infty}$ and $f$ an unbounded Bloch function, such that the product $g \cdot f$ is not a Bloch function.
\end{abstract}

\section{INTRODUCTION AND STATEMENTS OF THE RESUlts}

Let $\mathbb{D}=\{z \in \mathbb{C}:|z|<1\}$ denote the open unit disc in the complex plane $\mathbb{C}$. The space of all analytic functions in $\mathbb{D}$ will be denoted by $\mathcal{H o l}(\mathbb{D})$.

For $0<p \leq \infty$, the classical Hardy space $H^{p}$ is defined as the set of all $f \in \mathcal{H o l}(\mathbb{D})$ for which

$$
\|f\|_{H^{p}} \stackrel{\text { def }}{=} \sup _{0<r<1} M_{p}(r, f)<\infty
$$

where, for $0<r<1$ and $f \in \mathcal{H o l}(\mathbb{D})$,

$$
\begin{aligned}
M_{p}(r, f) & =\left(\frac{1}{2 \pi} \int_{0}^{2 \pi}\left|f\left(r e^{i \theta}\right)\right|^{p} d \theta\right)^{1 / p},(0<p<\infty) ; \\
M_{\infty}(r, f) & =\sup _{\theta \in \mathbb{R}}\left|f\left(r e^{i \theta}\right)\right| .
\end{aligned}
$$

We mention [7] as a general reference for the theory of Hardy spaces.

A function $f \in \mathcal{H}$ ol $(\mathbb{D})$ is said to be a Bloch function if

$$
\|f\|_{\mathcal{B}} \stackrel{\text { def }}{=}|f(0)|+\sup _{z \in \mathbb{D}}\left(1-|z|^{2}\right)\left|f^{\prime}(z)\right|<\infty .
$$

The space of all Bloch functions is denoted by $\mathcal{B}$, it is a Banach space with the just defined norm $\|\cdot\|_{\mathcal{B}}$. It is well known that

$$
H^{\infty} \subsetneq \mathcal{B} \text {. }
$$

Date: October 25, 2019.

1991 Mathematics Subject Classification. Primary 30D45; Secondary 30H30.

Key words and phrases. Bloch function, Normal function, Blaschke product, Inner function, Minimal Besov space, Analytic mean Lipschitz spaces.

This research is supported in part by a grant from "El Ministerio de Economía y Competitividad", Spain (PGC2018-096166-B-I00) and by grants from la Junta de Andalucía (FQM-210 and UMA18-FEDERJA-002). 
A typical example of an unbounded Bloch function is the function $f$ defined by

$$
f(z)=\log \frac{1}{1-z}, \quad z \in \mathbb{D} .
$$

We mention [1] as a general reference for the theory of Bloch functions.

A function $f$ which is meromorphic in $\mathbb{D}$ is said to be a normal function in the sense of Lehto and Virtanen [15] if

$$
\sup _{z \in \mathbb{D}}\left(1-|z|^{2}\right) \frac{\left|f^{\prime}(z)\right|}{1+|f(z)|^{2}}<\infty .
$$

For simplicity, we shall let $\mathcal{N}$ denote the set of all holomorphic normal functions in $\mathbb{D}$. It is clear that any Bloch function is a normal function, that is, we have $\mathcal{B} \subset \mathcal{N}$. We refer to [1], [15] and [16] for the theory of normal functions. In particular, we remark here that if $f \in \mathcal{N}, \xi \in \partial \mathbb{D}$ and $f$ has the asymptotic value $L$ at $\xi$, (that is, there exists a curve $\gamma$ in $\mathbb{D}$ ending at $\xi$ such that $f(z) \rightarrow L$, as $z \rightarrow \xi$ along $\gamma$ ) then $f$ has the non-tangential limit $L$ at $\xi$.

Let us recall that if a sequence of points $\left\{a_{n}\right\}$ in the unit disc satisfies the Blaschke condition: $\sum_{n=1}^{\infty}\left(1-\left|a_{n}\right|\right)<\infty$, the corresponding Blaschke product $B$ is defined as

$$
B(z)=\prod_{n=1}^{\infty} \frac{\left|a_{n}\right|}{a_{n}} \frac{a_{n}-z}{1-\overline{a_{n}} z} .
$$

Such a product is analytic in $\mathbb{D}$. In fact, it is an inner function, that is, an $H^{\infty}$-function with radial limit of absolute value 1 at almost every point of $\partial \mathbb{D}$ (cf. [7, Chapter 2]).

If $\left\{a_{n}\right\}$ is a Blaschke sequence and there exists $\delta>0$ such that

$$
\prod_{m \neq n}\left|\frac{a_{n}-a_{m}}{1-\bar{a}_{n} a_{m}}\right| \geq \delta, \quad \text { for all } n,
$$

we say that the sequence $\left\{a_{n}\right\}$ is uniformly separated and that $B$ is an interpolating Blaschke product. Equivalently,

$B$ is an interpolating Blaschke product $\Leftrightarrow \inf _{n \geq 1}\left(1-\left|a_{n}\right|^{2}\right)\left|B^{\prime}\left(a_{n}\right)\right|>0$.

We refer to [7, Chapter 9] and [9, Chapter VII] for the basic properties of interpolating Blaschke products. In particular, we recall that an exponential sequence is uniformly separated and that the converse holds if all the $a_{k}$ 's are positive.

Lappan [14, Theorem 3] proved that if $B$ is an interpolating Blaschke product and $f$ is a normal analytic function in $\mathbb{D}$, the product $B \cdot f$ 
need not be normal. Lappan used this to show that $\mathcal{N}$ is not a vector space.

Lappan's result is a consequence of the following easy fact: if $B$ is an interpolating Blaschke product whose sequence of zeros is $\left\{a_{n}\right\}$ and $G$ is an analytic function in $\mathbb{D}$ with $G\left(a_{n}\right) \rightarrow \infty$, then $f=B \cdot G$ is not a normal function (and hence it is not a Bloch function either). This result has been used by several authors (see [5, 17, 18, 10, 11, 3]) to construct distinct classes of non-normal functions.

The author and Suárez proved in [13] a result of this kind dealing with Blaschke products with zeros in a Stolz angle but not necessarily interpolating, improving a result of [12]. Namely, Theorem 1 of [13] is the following.

Theorem A. Let $B$ be an infinite Blaschke product whose sequence of zeros $\left\{a_{n}\right\}$ is contained in a Stolz angle with vertex at 1 and let $G$ be analytic in $\mathbb{D}$ with $G(z) \rightarrow \infty$, as $z \rightarrow 1$. Then the function $f=B \cdot G$ is not a normal function.

It is natural to ask whether it is possible to prove results similar to those described, substituting "Blaschke products" by some other classes of $H^{\infty}$-functions. Our first result in this paper deals with the atomic singular inner function.

Theorem 1.1. Let $S$ be the atomic singular inner function defined by

$$
S(z)=\exp \left(-\frac{1+z}{1-z}\right), \quad z \in \mathbb{D},
$$

and let $f$ be a Bloch function with

$$
\lim _{z \rightarrow 1}|f(z)|=\infty .
$$

Then the function $F$ defined by $F(z)=S(z) f(z)$ is not a normal function (hence, it is not a Bloch function).

In particular, the function $f$ defined by $f(z)=S(z) \cdot \log \frac{1}{1-z}(z \in \mathbb{D})$ is not normal. Since a Bloch function satisfies $M_{\infty}(r, f)=\mathrm{O}\left(\log \frac{1}{1-r}\right)$, Theorem 1.1 follows from the following result.

Theorem 1.2. Let $S$ be the singular inner function defined by $S(z)=$ $\exp \left(-\frac{1+z}{1-z}\right) \quad(z \in \mathbb{D})$ and let $f$ be an analytic function in $\mathbb{D}$ satisfying:

(i) $\lim _{z \rightarrow 1}|f(z)|=\infty$.

(ii) $|f(r)|=\mathrm{o}\left(\exp \frac{1+r}{1-r}\right)$, as $r \rightarrow 1^{-}$.

Then the function $F$ defined by $F(z)=S(z) f(z)$ is not a normal function (hence, it is not a Bloch function). 
For $g \in \mathcal{H o l}(\mathbb{D})$, the multiplication operator $M_{g}$ is defined by

$$
M_{g}(f)(z) \stackrel{\text { def }}{=} g(z) f(z), \quad f \in \mathcal{H} \text { ol }(\mathbb{D}), z \in \mathbb{D} .
$$

Let us recall that if $X$ and $Y$ are two spaces of analytic function in $\mathbb{D}$ and $g \in \mathcal{H} o l(\mathbb{D})$ then $g$ is said to be a multiplier from $X$ to $Y$ if $M_{g}(X) \subset Y$. The space of all multipliers from $X$ to $Y$ will be denoted by $M(X, Y)$ and $M(X)$ will stand for $M(X, X)$. Brown and Shields [4] characterized the space of multipliers of the Bloch space $M(B)$ as follows.

Theorem B. A function $g \in \mathcal{H} o l(\mathbb{D})$ is a multiplier of the Bloch space if and only if $g \in H^{\infty} \cap \mathcal{B}_{\text {log }}$, where $\mathcal{B}_{\log }$ is the Banach space of those functions $f \in \mathcal{H}$ ol $(\mathbb{D})$ which satisfy

$$
\|f\|_{B_{\log }} \stackrel{\text { def }}{=}|f(0)|+\sup _{z \in \mathbb{D}}\left(1-|z|^{2}\right)\left(\log \frac{2}{1-|z|^{2}}\right)\left|f^{\prime}(z)\right|<\infty .
$$

Thus, if $g \in H^{\infty} \backslash \mathcal{B}_{\log }$ there exists a function $f \in \mathcal{B} \backslash H^{\infty}$ such that $g \cdot f \notin \mathcal{B}$. It is easy to see that the analytic Lipschitz spaces $\Lambda_{\alpha}(0<\alpha \leq 1)$ and the mean Lipschitz spaces $\Lambda_{\alpha}^{p}(1<p<\infty$, $1 / p<\alpha \leq 1)$ are contained in $M(\mathcal{B})$ We refer to [7, Chapter 5] for the definitions of these spaces, let us simply recall here that

$$
\Lambda_{1}^{1}=\left\{f \in \mathcal{H o l}(\mathbb{D}): f^{\prime} \in H^{1}\right\} .
$$

On the other hand, Theorem 1 of [8] shows the existence of a Jordan domain $\Omega$ with rectifiable boundary and $0 \in \Omega$, and such that the conformal mapping $g$ from $\mathbb{D}$ onto $\Omega$ with $g(0)=0$ and $g^{\prime}(0)>0$ does not belong to $\mathcal{B}_{\log }$. For this function $g$ we have that $g \in \Lambda_{1}^{1}$ but $g$ is not a multiplier of $\mathcal{B}$. Thus we have:

$$
\Lambda_{1}^{1} \not \subset M(\mathcal{B}) .
$$

In view of this and the results involving Blaschke products that we have mentioned above, it is natural to ask the following question:

Question 1.3. Is it true that for any given $f \in \mathcal{B} \backslash H^{\infty}$ there exists a function $g \in \Lambda_{1}^{1}$ such that $g \cdot f \notin \mathcal{B}$ ?

We shall show that the answer to this question is affirmative. Actually we shall prove a stronger result.

We let $B^{1}$ denote the minimal Besov space which consists of those functions $f \in \mathcal{H}$ ol $(\mathbb{D})$ such that

$$
\int_{\mathbb{D}}\left|f^{\prime \prime}(z)\right| d A(z)<\infty .
$$


Here $d A$ denotes the area measure on $\mathbb{D}$. Alternatively, the space $B^{1}$ can be characterized as follows (see [2]):

For $f \in \mathcal{H o l}(\mathbb{D})$, we have that $f \in B^{1}$ if and only there exist a sequence of points $\left\{a_{k}\right\}_{k=1}^{\infty} \subset \mathbb{D}$ and a sequence $\left\{\lambda_{k}\right\}_{k=0}^{\infty} \in \ell^{1}$ such that

$$
f(z)=\lambda_{0}+\sum_{k=1}^{\infty} \lambda_{k} \varphi_{a_{k}}(z), \quad z \in \mathbb{D} .
$$

Here, for $a \in \mathbb{D}, \varphi_{a}: \mathbb{D} \rightarrow \mathbb{D}$ denotes the Möbius transformation defined by

$$
\varphi_{a}(z)=\frac{a-z}{1-\bar{a} z}, \quad z \in \mathbb{D} .
$$

is is well known that $B^{1} \subset \Lambda_{1}^{1}$ (see [2, 6]) and then our next result implies that the answer to question 1.3 is affirmative.

Theorem 1.4. If $f \in \mathcal{B} \backslash H^{\infty}$ then there exists $g \in B^{1}$ such that $g \cdot f \notin \mathcal{B}$.

The proofs of Theorem 1.2 and Theorem 1.4 will be presented in section2. We close this section noticing that throughout the paper we shall be using the convention that $C=C(p, \alpha, q, \beta, \ldots)$ will denote a positive constant which depends only upon the displayed parameters $p, \alpha, q, \beta \ldots$ (which often will be omitted) but not necessarily the same at different occurrences. Moreover, for two real-valued functions $E_{1}, E_{2}$ we write $E_{1} \lesssim E_{2}$, or $E_{1} \gtrsim E_{2}$, if there exists a positive constant $C$ independent of the arguments such that $E_{1} \leq C E_{2}$, respectively $E_{1} \geq C E_{2}$. If we have $E_{1} \lesssim E_{2}$ and $E_{1} \gtrsim E_{2}$ simultaneously then we say that $E_{1}$ and $E_{2}$ are equivalent and we write $E_{1} \asymp E_{2}$.

\section{THE PROOFS}

Proof of Theorem 1.2. For $0<a<1$, set $\Gamma_{a}=\{z \in \mathbb{D}:|z-a|=1-a\}$. If $z \in \Gamma_{a}$ then $\operatorname{Re} \frac{1+z}{1-z}=\frac{a}{1-a}$ and, hence,

$$
|S(z)|=\exp \left(\frac{-a}{1-a}\right), \quad z \in \Gamma_{a} .
$$

This, together with (i), implies that

$$
F(z) \rightarrow \infty, \quad \text { as } z \rightarrow 1 \text { along } \Gamma_{a} .
$$

Hence $F$ has the asymptotic value $\infty$ at 1 . On the other hand, (ii) implies that $F$ has the radial limit 0 at 1 . Then it follows that $F$ is not normal. 
Proof of Theorem 1.4. Take $f \in \mathcal{B} \backslash H^{\infty}$. Set

$$
\varphi(r)=M_{\infty}(r, g), \quad 0<r<1 .
$$

Clearly, $\varphi(r) \rightarrow \infty$, as $r \rightarrow 1$ and it is well known that

$$
\phi(r)=\mathrm{O}\left(\log \frac{1}{1-r}\right) .
$$

This implies that

$$
(1-r)^{2} \varphi(r) \rightarrow 0, \quad \text { as } r \rightarrow 1 .
$$

Choose a sequence of numbers $\left\{r_{n}\right\} \subset(0,1)$ satisfying the following properties:

(i) $\left\{r_{n}\right\}$ is increasing.

(ii) $\left(1-r_{n}\right)^{2} \varphi\left(r_{n}\right)=\mathrm{o}\left(\left(\frac{1-r_{n-1}}{n}\right)^{2}\right), \quad$ as $n \rightarrow \infty$.

(iii) $\varphi\left(r_{n}\right) \geq 2 \varphi\left(r_{n-1}\right)$, for all $n$.

(iv) $\frac{1-r_{n+1}}{1-r_{n}} \rightarrow 0$, as $n \rightarrow \infty$.

The existence of such a sequence is clear, bearing in mind (2.1) and the the fact that $\varphi(r) \rightarrow \infty$, as $r \rightarrow 1$.

Set

$$
\lambda_{k}=\varphi\left(r_{k}\right)^{-1 / 2}, \quad k=1,2, \ldots
$$

For each $k$, take $a_{k} \in \mathbb{D}$ with $\left|a_{k}\right|=r_{k}$ and $\left|f\left(a_{k}\right)\right|=\varphi\left(r_{k}\right)$. Using (iii), it follows that

$$
\sum_{k=1}^{\infty} \lambda_{k}<\infty
$$

Define

$$
g(z)=\sum_{k=1}^{\infty} \lambda_{k} \varphi(z), \quad z \in \mathbb{D} .
$$

Using (2.2) we see that the sum in (2.3) defines an analytic function in $\mathbb{D}$ which belongs to $B^{1}$. Set

$$
F(z)=g(z) f(z), \quad z \in \mathbb{D} .
$$

Since $g \in H^{\infty}$ and $f \in \mathcal{B}$ we see that

$$
\left|g\left(a_{n}\right) f^{\prime}\left(a_{n}\right)\right|=\mathrm{O}\left(\frac{1}{1-\left|a_{n}\right|}\right) .
$$

On the other hand,

$$
\left|g^{\prime}\left(a_{n}\right) f\left(a_{n}\right)\right| \gtrsim I-I I-I I I,
$$


where

$$
\begin{aligned}
I & =\left|f\left(a_{n}\right)\right| \lambda_{n}\left|\varphi_{a_{n}}\left(a_{n}\right)\right|, \quad I I \lesssim\left|f\left(a_{n}\right)\right| \sum_{k=1}^{n-1} \lambda_{k} \frac{1-\left|a_{k}\right|^{2}}{\left|1-\overline{a_{k}} a_{n}\right|^{2}}, \\
I I I & \lesssim\left|f\left(a_{n}\right)\right| \sum_{k=n+1}^{\infty} \lambda_{k} \frac{1-\left|a_{k}\right|^{2}}{\left|1-\overline{a_{k}} a_{n}\right|^{2}} .
\end{aligned}
$$

Clearly,

$$
I=\left|f\left(a_{n}\right)\right| \lambda_{n}\left|\varphi_{a_{n}}\left(a_{n}\right)\right| \asymp \frac{\varphi\left(r_{n}\right)^{1 / 2}}{1-r_{n}} .
$$

Using the definitions, the facts that $\varphi$ and the sequence $\left\{r_{n}\right\}$ are increasing, and (ii), we obtain

$$
\begin{aligned}
I I & \lesssim\left|f\left(a_{n}\right)\right| \sum_{k=1}^{n-1} \lambda_{k} \frac{1-\left|a_{k}\right|^{2}}{\left|1-\overline{a_{k}} a_{n}\right|^{2}} \\
& \lesssim \varphi\left(r_{n}\right) \sum_{k=1}^{n-1} \varphi\left(r_{k}\right)^{-1 / 2} \frac{1-\left|a_{k}\right|}{\left[\left(1-\left|a_{k}\right|\right)+\left(1-\mid a_{n}\right)\right]^{2}} \\
& \lesssim \varphi\left(r_{n}\right) \sum_{k=1}^{n-1} \frac{1}{\varphi\left(r_{k}\right)^{1 / 2}\left(1-r_{k}\right)} \\
& \lesssim \frac{n \varphi\left(r_{n}\right)}{1-r_{n-1}} \\
& =\frac{\varphi\left(r_{n}\right)^{1 / 2}}{1-r_{n}} \varphi\left(r_{n}\right)^{1 / 2} \frac{n\left(1-r_{n}\right)}{1-r_{n-1}} \\
& =\mathrm{o}\left(\frac{\varphi\left(r_{n}\right)^{1 / 2}}{1-r_{n}}\right) .
\end{aligned}
$$


Likewise, using the definitions, the facts that $\varphi$ and the sequence $\left\{r_{n}\right\}$ are increasing, (iii), and (iv), we obtain

$$
\begin{aligned}
I I I & \lesssim \varphi\left(r_{n}\right) \sum_{k=n+1}^{\infty} \frac{\varphi\left(r_{k}\right)^{-1 / 2}\left(1-r_{k}\right)}{\left[\left(1-r_{k}\right)+\left(1-r_{n}\right)\right]^{2}} \\
& \lesssim \varphi\left(r_{n}\right) \sum_{k=n+1}^{\infty} \varphi\left(r_{k}\right)^{-1 / 2} \frac{1-r_{k}}{\left(1-r_{n}\right)^{2}} \\
& \lesssim \varphi\left(r_{n}\right) \frac{1-r_{n+1}}{\left(1-r_{n}\right)^{2}} \sum_{k=n+1}^{\infty} \varphi\left(r_{k}\right)^{-1 / 2} \\
& \lesssim \frac{\varphi\left(r_{n}\right)^{1 / 2}}{1-r_{n}} \cdot \frac{1-r_{n+1}}{1-r_{n}} \\
& =\mathrm{o}\left(\frac{\varphi\left(r_{n}\right)^{1 / 2}}{1-r_{n}}\right) .
\end{aligned}
$$

Using (2.6), (2.7), (2.8), and the fact that $\lim \varphi\left(r_{n}\right)=\infty$, we deduce that

$$
\left(1-\left|a_{n}\right|\right)\left|g^{\prime}\left(a_{n}\right) f\left(a_{n}\right)\right| \rightarrow \infty, \quad \text { as } n \rightarrow \infty .
$$

This and (2.4) imply that $F$ is not a Bloch function.

\section{REFERENCES}

1. J. M. Anderson, J. Clunie and Ch. Pommerenke, On Bloch functions and normal functions, J. Reine Angew. Math. 270 (1974), 12-37.

2. J. Arazy, S. D. Fisher and J. Peetre, Möbius invariant function spaces, J. Reine Angew. Math. 363 (1985), 110-145.

3. O. Blasco, D. Girela and M. A. Márquez, Mean growth of the derivative of analytic functions, bounded mean oscillation, and normal functions, Indiana Univ. Math. J. 47 (1998), 893-912.

4. L. Brown and A. L. Shields, Multipliers and cyclic vectors in the Bloch space, Michigan Math. J. 38 (1991), no. 1, 141-146.

5. D. M. Campbell, Nonnormal sums and products of unbounded normal functions. II., Proc. Amer. Math. Soc. 74 (1979), no. 1, 202-203.

6. J. J. Donaire, D. Girela and D. Vukotić, On univalent functions in some Möbius invariant spaces, J. Reine Angew. Math. 553 (2002), 43-72.

7. P. L. Duren, Theory of $H^{p}$ spaces, Academic Press, New York-London, 1970. Reprint: Dover, Mineola-New York, 2000.

8. P. Galanopoulos, D. Girela and R. Hernández, Univalent functions, VMOA and related spaces J. Geom. Anal. 21 (2011), no. 3, 665-682.

9. J. B. Garnett, Bounded Analytic Functions, Academic Press, New York, etc. (1981).

10. D. Girela, On a theorem of Privalov and normal funcions, Proc. Amer. Math. Soc. 125, no. 2 (1997), 433-442. 
11. D. Girela, Mean Lipschitz spaces and bounded mean oscillation, Illinois J. Math. 41, No. 2 (1997), 214-230.

12. D. Girela, C. González and J. A. Peláez, Multiplication and division by inner functions in the space of Bloch functions, Proc. Amer. Math. Soc. 134, No. 5 (2006), 1309-1314.

13. D. Girela and D. Suárez, On Blaschke products, Bloch functions and normal functions, Rev. Mat. Complut. 24 (2011), no. 1, 49-57.

14. P. Lappan Non-normal sums and products of unbounded normal function, Michigan Math. J. 8 (1961), 187-192.

15. O. Lehto and K. I. Virtanen, Boundary behaviour and normal meromorphic functions, Acta Math. 97 (1957), 47-65.

16. Ch. Pommerenke, Univalent Functions, Vandenhoeck und Ruprecht, Göttingen, (1975).

17. S. Yamashita, A nonnormal function whose derivative has finite area integral of order $0<p<2$, Ann. Acad. Sci. Fenn. Ser. A I Math. 4 (1979), no. 2, 293-298.

18. S. Yamashita, A nonnormal function whose derivative is of Hardy class $H^{p}$, $0<p<1$, Canad. Math. Bull. 23 (1980), no. 4, 499-500.

Análisis Matemático, Facultad de Ciencias, Universidad de Málaga, 29071 Málaga, Spain

E-mail address: girela@uma.es 